\title{
STOCHASTIC CONSTRUCTION OF NEW ORTHOGONAL POLYNOMIALS
}

\author{
T. F. LIN
}

ABSTRACT. For $u>-1, t>0, x \in R$, let

$$
(1+u)^{x} e^{-u t}=\sum_{n=0}^{\infty} u^{n} K_{n}(t, x)
$$

Then

(i) $(1+u)^{Q(t)+t} e^{-u t}$ satisfies $d y(t)=u y(t) d Q(t)$ where $Q(t)=P(t)-t$

is the centered Poisson process with parameter $\lambda=1$,

(ii) $K_{n}(t, x), n>0$, is a system of complete orthogonal polynomials in

$L^{2}(R, d F)$ where $F(x)$ is the distribution function of $P(t)$,

(iii) $(n+1) K_{n+1}(t, x)-(x-t-n) K_{n}(t, x)+t K_{n-1}(t, x)=0, n>1$,

(iv) $t \Delta^{2} K_{n}(t, x)+(t-x) \Delta K_{n}(t, x)+n K_{n}(t, x)=0, n>0$.

1. Introduction. The usual way to prove the orthogonality of orthogonal systems like Legendre polynomials, Laguerre polynomials or Hermite polynomials is to use the fact that these polynomials can be obtained by differentiating certain respective functions (see [2, pp. 92-94]). In this article, we shall construct a system of polynomials and prove their orthogonality by using the probabilistic method. This system of polynomials will also satisfy some recursive formulas and difference equations as those polynomials mentioned above do. The motivation comes from the following observation. Let $I_{W}(f)(t)$ denote Ito's integral of the Brownian nonanticipating functional $f(t)$ w.r.t. the standard Brownian motion $W(t)$ over $[0, t]$ and let $I_{W}^{n}(f)(t)=$ $I_{W}\left(I_{W}^{n-1}(f)\right)(t), n>1$. Then

PROPERTY 1.1. $E\left\{I_{W}(f)(t)\right\}=0$,

$$
E\left\{I_{W}(f)(t) I_{W}(g)(t)\right\}=\int_{0}^{t} E\{f(s) g(s)\} d s
$$

if each term appearing above is well defined.

PROPERTY 1.2. $I_{W}^{n}(1)(t), n>0$, is a polynomial of $W(t)$. Indeed, $I_{W}^{n}(1)(t)=$ $H_{n}(t, W(t))$ where $H_{n}(t, x)$ is the $n$th Hermite polynomial (see [5, p. 38]).

Combining Properties 1.1 and 1.2, it is easily seen that $H_{n}(t, x), H_{m}(t, x)$, $n \neq m$, are orthogonal in $L^{2}(R, d F)$ where

$$
d F(x)=(2 \pi t)^{-1 / 2} \exp \left(-x^{2} / 2 t\right) d x .
$$

Received by the editors February 27, 1978.

AMS (MOS) subject classifications (1970). Primary 42A52, 60H99; Secondary 39A10, 33A65. Key words and phrases. Orthogonal polynomials, stochastic integral, difference equation. 
If we can replace $W(t)$ in Properties 1.1 and 1.2 by some other process $X(t)$, then we obtain another system of polynomials which are orthogonal w.r.t. the distribution function (measure) of $X(t)$. We shall see that the centered Poisson process is one of this kind of $X(t)$.

Let $P(t), t \geqslant 0$, be a Poisson process with parameter $\lambda=1$. Then $(\Delta P(t))^{m}$ $\doteq \Delta P(t)$ for $m>1$. This comes as no surprise since

$$
P\left\{(\Delta P(t))^{m} \neq \Delta P(t)\right\}=O\left((\Delta t)^{2}\right) .
$$

However, it is striking. By using this fact, it was proved in Theorem 2.3 of [4], that for each $u>-1, Y(t)=(1+u)^{P(t)}$ is the solution of the stochastic equation

$$
Y(t)=Y(s)+\int_{s}^{t} u Y(r) d P(r), \quad 0 \leqslant s \leqslant t .
$$

We shall make use of (1.1) to derive our main result, Theorem 2.3.

2. Integration. Let $Q(t)=P(t)-t, t \geqslant 0$, denote the centered Poisson process with Parameter $\lambda=1$. We want to define the integral w.r.t. $Q(t)$. Instead of using the martingale integral (see [3]) or the belated integral (see [6]), we shall use the simplest Cauchy definition of integral since the latter is good enough for our purpose. For the integrand $f(t)$, we always assume the following conditions.

(2.1) For $s<t, P(t)-P(s)$ is independent of $\{f(r), P(r) ; r \leqslant s\}$.

(2.2) Almost surely, the sample paths of $f(t)$ have no second kind of discontinuity.

If $f(t)$ satisfies (2.2), then the following integral is well defined.

$$
\begin{aligned}
I_{P}(f)(t) & =\int_{0}^{t} f(s) d P(s) \\
& =\lim _{n \rightarrow \infty} \sum_{0<k<2^{n}} f\left(t_{k}\right)\left\{P\left(t_{k+1}\right)-P\left(t_{k}\right)\right\}
\end{aligned}
$$

where $t_{k}=k t / 2^{n}, 0 \leqslant k \leqslant 2^{n}$. The limit in (2.3) is an a.e. limit. If $f(t)$ is $L^{1}$-continuous in $t$, then the convergence is also an $L^{1}$-convergence. From (2.1), it is easy to see that

$$
E\left\{I_{P}(f)(t)\right\}=\int_{0}^{t} E\{f(s)\} d s .
$$

Now define the integral of $f(t)$ w.r.t. $Q(t)$ as

$$
\begin{aligned}
I_{Q}(f)(t) & =\lim _{n \rightarrow \infty} \sum_{0<k<2^{n}} f\left(t_{k}\right)\left\{Q\left(t_{k+1}\right)-Q\left(t_{k}\right)\right\} \\
& =I_{P}(f)(t)-\int_{0}^{t} f(s) d s .
\end{aligned}
$$

Then $I_{Q}(f)(t)$ satisfies (2.1) and (2.2) automatically. Hence $I_{Q}^{2}(f)(t)=$ $I_{Q}\left(I_{Q}(f)\right)(t)$ is well defined. Similarly, $I_{Q}^{n}(f)=I_{Q}\left(I_{Q}^{n-1}(f)\right)(t), n \geqslant 3$, can be 
defined. Since $Q(t)$ has independent increments, it is trivial that

LEMMA 2.1. $E\left\{I_{Q}(f)(t)\right\}=0$,

$$
E\left\{I_{Q}(f)(t) I_{Q}(g)(t)\right\}=\int_{0}^{t} E\{f(s) g(s)\} d s
$$

whenever each term appearing above is defined.

For convenience, lẹt $I_{Q}^{0}(t)=1, I_{Q}^{n}(t)=I_{Q}^{n}(1)(t), n \geqslant 1, t \geqslant 0$. It follows from Lemma 2.1 that

COROLLARY 2.2. $I_{Q}^{n}(t), n \geqslant 0$, are orthogonal in $L^{2}(\Omega)$ and

$$
E\left\{\left(I_{Q}^{n}(t)\right)^{2}\right\}=t^{n} / n !
$$

Lemma 2.1 is equivalent to Property 1.1. To find orthogonal polynomials associated with $Q(t)$, we need to show that $I_{Q}^{n}(t)$ is a polynomial of $Q(t)$. To this end, let $Y(t)=(1+u)^{P(t)}, t \geqslant 0, u>-1$, be as in $\S 1$ which satisfied equation (1.1). After some routine manipulation, we obtain, for $0 \leqslant s \leqslant t \leqslant$ $T$,

$$
\begin{aligned}
Y(t) e^{-u t}-Y(s) e^{-u s} & =u Y(s) e^{-u s}\{P(t)-P(s)-t+s\}+R \\
& =u Y(s) e^{-u s}\{Q(t)-Q(s)\}+R
\end{aligned}
$$

where

$$
\begin{aligned}
R= & (Y(t)-Y(s))\left(e^{-u t}-e^{-u s}\right) \\
& +Y(s)\left(e^{-u t}-e^{-u s}+u(t-s) e^{-u s}\right) \\
& +u e^{-u s} \int_{s}^{t}(Y(r)-Y(s)) d P(r) .
\end{aligned}
$$

It is easy to check that $E\{Y(t)\} \leqslant e^{|u| t}$. From this and (2.4), we see that $E|R| \leqslant c(u, T)(t-s)^{2}$ where $c(u, T)$ depends only on $u$ and $T$. Set $t=(k$ $+1) t / 2^{n}, s=k t / 2^{n}$ in (2.6), sum over all $k \leqslant 2^{n}$ and let $n \rightarrow \infty$. We have, after using definition (2.5),

THEOREM 2.3. Let $u>-1, t$ in compact set. The process $Z(t)=Y(t) e^{-u t}$ satisfies the stochastic integral equation

$$
Z(t)=1+\int_{0}^{t} u Z(s) d Q(s)
$$

REMARK. By using $(\Delta Q(t))^{m} \doteq(\Delta P(t))^{m}+O\left((\Delta t)^{2}\right) \doteq \Delta P(t)=\Delta Q(t)+$ $\Delta t, m \geqslant 2$, and the same method used in deriving (1.1) (see [4]), we can derive that, for $c, r \in R, t \geqslant 0$,

$$
\begin{aligned}
e^{r Q(t)-c t}=1 & +\int_{0}^{t}\left(e^{r}-1\right) e^{r Q(s)-c s} d Q(s) \\
& +\int_{0}^{t}\left(e^{r}-1-r-c\right) e^{r Q(s)-c s} d s .
\end{aligned}
$$


Let $c=e^{r}-1-r, u=e^{r}-1$. Then the above equation becomes (2.7).

Theorem 2.3 will enable us to find $I_{Q}^{n}(t)$. Let $X(t)=\sum_{n=0}^{\infty} u^{n} I_{Q}^{n}(t)$ for $u>-1, t \geqslant 0$. Corollary 2.2 implies that $E\{X(t)\}^{2}=e^{t u^{2}}$. Hence $X(t)$ is the $L^{2}$-limit of $\sum_{n=0}^{m} u^{n} I_{Q}^{n}(t)$ as $m \rightarrow \infty$. It is trivial that $X(t)$ also satisfies equation (2.7). A standard argument (e.g. see [1, pp. 106-110]) shows that equation (2.7) has a unique solution. Therefore, $X(t)=Z(t)$ and

$$
(1+u)^{P(t)} e^{-u t}=\sum_{n=0}^{\infty} u^{n} I_{Q}^{n}(t) .
$$

Since $P(t)=Q(t)+t,(2.8)$ leads to

Corollary 2.4. For $n \geq 0$,

$$
I_{Q}^{n}(t)=\left\{\partial^{n} / \partial u^{n}(1+u)^{Q(t)+t} e^{-u t}\right\}_{u=0} / n !
$$

is a polynomial of $Q(t)$ of degree $n$.

3. Polynomials. For $u>-1, t \geqslant 0, x \in R$, let $h(u, t, x)=(1+u)^{x} e^{-u t}$ be the generating function of $K_{n}(t, x), n \geqslant 0$, that is,

$$
h(u, t, x)=\sum_{n=0}^{\infty} u^{n} K_{n}(t, x) .
$$

Then, for each $n \geqslant 0$,

$$
K_{n}(t, x)=\frac{1}{n !}\left\{\partial^{n} / \partial u^{n} h(u, t, x)\right\}_{u=0} .
$$

From (2.9) and (3.1), we see that for $n \geqslant 0$,

$$
I_{Q}^{n}(t)=K_{n}(t, P(t))=K_{n}(t, Q(t)+t) .
$$

Let $F(x)$ be the distribution function of $P(t)$. Then Corollary 2.2 and (3.2) imply that

Lemma 3.1 (ORTHOGONALITY). $K_{n}(t, x), n \geqslant 0$, is a family of polynomials orthogonal w.r.t. the measure $d F(x)$.

Let us now show that $K_{n}(t, x), n \geqslant 0$, satisfy certain recursive formulas and some difference equations. From (3.1),

$$
\begin{aligned}
(n+1) K_{n+1}(t, x) & =\frac{1}{n !}\left\{\partial^{n} / \partial u^{n}\{\partial / \partial u h(u, t, x)\}\right\}_{u=0} \\
& =\frac{1}{n !}\left\{\partial^{n} / \partial u^{n}\left\{x(1+u)^{x-1} e^{-u t}-t(1+u)^{x} e^{-u t}\right\}\right\}_{u=0} \\
& =\frac{1}{n !}\left\{x \partial^{n} / \partial u^{n} h(u, t, x-1)-t \partial^{n} / \partial u^{n} h(u, t, x)\right\}_{u=0}
\end{aligned}
$$

Therefore, again by (3.1), we obtain the identity

$$
(n+1) K_{n+1}(t, x)=x K_{n}(t, x-1)-t K_{n}(t, x) .
$$

From the definition of $h(u, t, x)$, it is easy to check that

$$
h(u, t, x+1)-h(u, t, x)=u h(u, t, x) .
$$


Comparing the coefficient of $u^{n+1}, n \geqslant 0$, we get another identity

$$
K_{n+1}(t, x+1)-K_{n+1}(t, x)=K_{n}(t, x) .
$$

With the help of the identities (3.3) and (3.4), we can derive

LemMa 3.2 (ReCURSIVE). For each $n \geqslant 1, t \geqslant 0, x \in R$,

$$
(n+1) K_{n+1}(t, x)-(x-t-n) K_{n}(t, x)+t K_{n-1}(t, x)=0 .
$$

Let $\Delta K_{n}(t, x)=K_{n}(t, x)-K_{n}(t, x-1), \Delta^{2} K_{n}(t, x)=\Delta K_{n}(t, x+1)-$ $\Delta K_{n}(t, x)$. Again, by using identities (3.3) and (3.4), it is not hard to derive

Lemma 3.3 (Difference). For $n \geqslant 0, t \geqslant 0, x \in R$,

$$
t \Delta^{2} K_{n}(t, x)-(x-t) \Delta K_{n}(t, x)+n K_{n}(t, x)=0 .
$$

4. Completeness. To see the completeness of the system $K_{n}(t, x), n \geqslant 0$, in $L^{2}(R, d F)$, we use the argument which was suggested by von Neumann. Let $K_{n}^{*}(t, y)$ denote the restriction of $K_{n}(t, \cdot)$ to $N=\{0,1,2, \ldots\}$. Since $d F$ concentrates its mass on $N$, the completeness of $K_{n}(t, x), n \geqslant 0$, in $L^{2}(R, d F)$ is equivalent to the completeness of $K_{n}^{*}(t, y), n \geqslant 0$, in $L^{2}\left(N, d F^{*}\right)$ where $d F^{*}$ is the restriction of $d F$ on the subsets of $N$. We shall prove the latter instead of the former. Let $H$ be the subspace of $L^{2}\left(N, d F^{*}\right)$ generated by $K_{n}^{*}(t, y)$, $n \geqslant 0$.

LeMma 4.1. For each $n \geqslant 0$, let $e_{n}(y)=e^{-n y}, y \in N$. Then $e_{n} \in H$.

Proof. Let $u>-1$ be arbitrary but fixed and let

$$
h_{n}(t, x)=\sum_{m=0}^{n} u^{m} K_{m}(t, x), \quad h_{n}^{*}(t, y)=\sum_{m=0}^{n} u^{m} K_{m}^{*}(t, y)
$$

Then (2.8) and (3.2) imply that $(1+u)^{P(t)} e^{-u t}=h(u, t, P(t))$ is the $L^{2}$-limit of $h_{n}(t, P(t))$ as $n \rightarrow \infty$. Let $h^{*}(u, t, y)$ denote the restriction of $h(u, t, \cdot)$ to $N$. Then $h^{*}(u, t, y)$ is the limit of $h_{n}^{*}(t, y)$ in $L^{2}\left(N, d F^{*}\right)$ as $n \rightarrow \infty$. Therefore, $h^{*}(u, t, y) \in H$. Thus $(1+u)^{y} \in H$ for each $u>-1$. Write $a=1+u>0$. Then $a^{y} \in H$ for each $a>0$. Lemma 4.1 follows by letting $a=e^{-n}$.

LEMMA 4.2. $H=L^{2}\left(N, d F^{*}\right)$.

Proof. For each $f \in L^{2}\left(N, d F^{*}\right)$, let $f_{n}=f \cdot I_{[0, n]}$ where $I_{[0, n]}$ denotes the indicator function of $[0, n]$. Then $f_{n}$ converges to $f$ in $L^{2}\left(N, d F^{*}\right)$ as $n \rightarrow \infty$. Let $M=\left\{e^{-n} ; n \in N\right\}$ and let $\dot{G}\left(\left\{e^{-n}\right\}\right)=F^{*}(\{n\})$ be the measure on subsets of $M$ induced by $F^{*}$. Then

$$
g_{n}(z)=f_{n}(-\log z) \in L^{2}(M, d G) .
$$

$g_{n}(z)$ can be continuously extended to $[0,1]$ such that $g_{n}(0)=0$. There exists polynomials $p_{m}^{(n)}(z)$ converging uniformly to $g_{n}(z)$ on $[0,1]$ as $m \rightarrow \infty$. From 
Lemma $4.1, p_{m}^{(n)}\left(e^{-y}\right) \in H$. Since

$$
\begin{aligned}
\int_{N}\left|f_{n}(y)-p_{m}^{(n)}\left(e^{-y}\right)\right|^{2} d F^{*}(y) & <\sup _{0<z<1}\left|f_{n}(-\log z)-p_{m}^{(n)}(z)\right|^{2} \\
& \leqslant \sup _{0<z<1}\left|g_{n}(z)-p_{m}^{(n)}(z)\right|^{2},
\end{aligned}
$$

we see that $f_{n} \in H$ after letting $m \rightarrow \infty$. Therefore $f \in H$. This proves Lemma 4.2.

Lemma 4.2 is equivalent to the statement that $L^{2}(R, d F)$ is generated by $K_{n}(t, x), n \geqslant 0$. This fact together with Lemma 3.1 lead to

THEOREM 4.3. The family of polynomials $K_{n}(t, x), n \geqslant 0$, is a complete orthogonal system in $L^{2}(R, d F)$.

\section{BIBLIOGRAPHY}

1. L. Arnold, Stochastic differential equations, Wiley, New York, 1974.

2. R. Courant and D. Hilbert, Methods of mathematical physics. I, Interscience, New York, 1955.

3. H. Kunita and S. Watanabe, On square integrable martingales, Nagoya Math. J. 30 (1967), 209-245.

4. T. F. Lin, An identity for multiple integral of Poisson process, Soochow J. Math. Nat. Sci. 3 (1977), 35-40.

5. H. P. McKean, Jr., Stochastic integrals, Academic Press, New York, 1969.

6. E. J. McShane, Stochastic calculus and stochastic models, Academic Press, New York, 1974.

Department of Business Mathematics, Soochow University, Taipei, Tarwan, China 